\title{
Bi
}

UNIVERSIDAD DEL PACÍFICO

\section{Estrategias de mujeres ejecutivas exitosas}

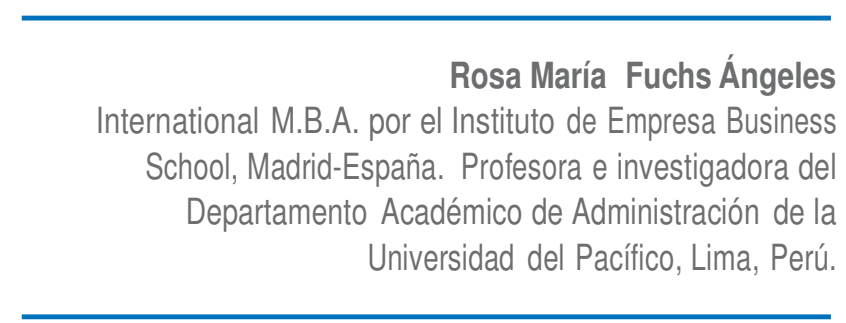

\section{Resumen}

En Latinoamérica es aún muy limitado el porcentaje de mujeres que logra superar la línea media de las empresas y llegar a ocupar puestos de dirección. La búsqueda de igualdad de oportunidades para hombres y mujeres es una preocupación a nivel mundial y si bien se trabaja en esa dirección, las brechas aún existen. En este artículo se explora la realidad de la mujer ejecutiva en la empresa sobre la base de diversos estudios que se han realizado sobre el tema y se realiza un acercamiento a la realidad de mujeres ejecutivas exitosas en el Perú para poder definir un perfil sobre ellas y descubrir la estrategias que han seguido en el desarrollo de su línea de carrera.

\section{Palabras clave}

Mujer ejecutiva, desarrollo de carrera, estrategia.

\section{Introducción}

Las mujeres ocupan un lugar importante en la fuerza de trabajo latinoamericana. Sin embargo, los porcentajes indican que una minoría de los puestos directivos es ocupada por mujeres. A nivel mundial, las mujeres ocupan 1 de cada 5 puestos gerenciales (Grant Thornton 2012). En América Latina, un artículo denominado Tierra de Hombres (García 2010), da cuenta de que en los consejos de administración de las 100 empresas más grandes de América Latina, sólo el $7 \%$ de 654 asientos disponibles es ocupado por mujeres. Como dato adicional, el autor comenta que muchas de estas mujeres pertenecen a las familias que controlan a estas empresas. 
The Corporate Gender Gap Report (World Economic Forum 2010) muestra los resultados de encuestas aplicadas a los 100 más grandes empleadores decadauno delos30paísesmiembros de la OCDE (Organización para la Cooperación y el Desarrollo Económico) incluyendo a Brasil, Rusia, India y China. Según este informe las mujeres se concentran en posiciones iniciales o de línea media en las empresas. En promedio, en todos los países encuestados, las mujeres en la posición de CEO son un poco menos que el $5 \%$. La cifra antes mencionada está altamente relacionada con lo que presenta el listado de empresas Fortune 500, pues en él, el porcentaje de mujeres CEO es sólo el 3\% (Fortune 2010).

Las ejecutivas exitosas que han logrado desarrollar una línea de carrera competitiva han seguido estrategias para conseguirlo. Es la intención de este estudio profundizar en dichas estrategias. El presente documento parte de los estudios referidos a la situación de la mujer que trabaja, posteriormente se profundiza en las estrategias de desarrollo de carrera que ejecutivas exitosas han aplicado y se presentan las conclusiones respectivas.

\section{Objetivo de la investigación}

- Identificar estrategias de desarrollo de línea de carrera que fueron exitosas para ejecutivas en el contexto peruano.

\section{Revisión de literatura}

La OIT (2007), en su informe titulado La igualdad en el trabajo: afrontar los retos que se plantean, analiza la evolución de una serie de factores a nivel mundial con el único fin de lograr la igualdad de oportunidades laborales. En líneas generales, en cuanto a género se refiere, las mujeres siguen ganando menos y se encuentran en desventaja para un empleo a tiempo completo. Este informe nos revela que en el mundo ya se hace referencia al trabajo «idéntico» pero no de «igual valor». Es importante mencionar que según este informe no se considera discriminación el aplicar medidas especiales que conllevan a un trato diferenciado para quienes tienen necesidades particulares. Otro informe de la OIT (2010), titulado Women in labour markets: Measuring progress and identifying challenges señala que hace quince años se inició en Beijing el esfuerzo por proponer una plataforma para la igualdad de género. Algunos de los objetivos que se perseguían eran el acceso al empleo, las condiciones de trabajo apropiadas, eliminar la discriminación en el trabajo, armonía entre el trabajo y vida familiar para hombres y mujeres. El esfuerzo continúa y se enfatiza que no se debe ver a la mujer en el mundo de los negocios de los hombres sino que el mundo de los negocios es el que debe adaptarse a las características de la mujer. Según este informe el $48 \%$ de la mujeres en edad de trabajar se encuentra empleada y en el caso de los hombres es el $72.8 \%$ en el año 2009. El informe presenta 12 indicadores llamados Key Indicators of the Labour Market, los que analizan la evolución de la igualdad de género.

El indicador sobre empleo por sector nos muestra que las mujeres dominan los sectores de servicio doméstico, salud, trabajo social, educación, hoteles y restaurantes, trabajos comunitarios, intermediación financiera en las economías desarrolladas. En general el trabajo femenino se concentra en servicios, caracterizados por bajos pagos, largas jornadas de trabajo y arreglos informales de trabajo. Aún en estos sectores es raro encontrar a mujeres en posiciones gerenciales.

Claudia Anzorena (Anzorena 2008) menciona que el mundo del trabajo se percibe como el mundo masculino y el mundo doméstico como el de las mujeres. La esfera privada connota lo femenino como lo relacionado con sentimientos y debilidad y la esfera pública connota lo masculino como lo relacionado a razón y fuerza. Anzorena trata de explicar las diferencias entre mujeres y hombres en el mercado laboral refiriéndose a que el trabajo de la mujer se considera secundario respecto de su papel reproductor y subordinado en relación a un ingreso masculino privilegiado. Un factor que se menciona en este artículo es que las mujeres occidentales participan más en el ámbito público pero esto no significa que se hayan desligado de parte de sus tareas en el ámbito doméstico. Las mujeres siguen siendo consideradas las responsables de las tareas domésticas en el hogar 
y, en la esfera laboral siguen ubicándose en los ámbitos menos valorados.

Igualmente, Lieve Daeren (2000) menciona que las mujeres deben combinar dos jornadas de trabajo, la del mundo laboral y la del hogar. Sostiene que las sociedades son las que no asumen la responsabilidad de la maternidad y el cuidado de las personas, consideran que esta es una responsabilidad individual y esto hace que las mujeres partan en condiciones desiguales. Según Daeren las mujeres quieren desempeñar el trabajo de los hombres pero los hombres no desean el de las mujeres por ser considerados de menor paga y menos valorizados.

Como causas de la discriminación sexista, el artículo La Discriminación en el trabajo de Newell (2002) nos presenta dos: el doble papel de la mujer (debe ser buena madre y buena trabajadora), los estereotipos y actitudes organizacionales (se cree que la mujer es menos enérgica, menos ambiciosa y que tiene menos interés en su carrera). La mujer lucha entonces no solo con sus dobles responsabilidades sino con lo que la gente que trabaja con ella piensa que deben ser sus responsabilidades. Según este artículo las mujeres directivas atribuyen su éxito al entorno y a la suerte más que los directivos varones, y se lo atribuyen menos a su capacidad. Esta modestia inhibe el progreso femenino pero es parte de los prejuicios en los que las mismas mujeres viven.

Una encuesta de Catalyst (2009), sobre las mujeres que dejan y vuelven a la fuerza laboral en Estados Unidos, revela un dato interesante. Se cree que las mujeres dejan su trabajo fácilmente para quedarse en casa con sus hijos. Sin embargo, el estudio revela que las mujeres sienten grandes conflictos al dejar su trabajo y lo encuentran muy difícil. La razón es que han invertido mucho esfuerzo en su preparación profesional y el trabajo es realmente importante en sus vidas. Si dejan el trabajo no es por tener hijos sino porque los empleadores no les brindan las facilidades para combinar el trabajo y el resto de su vida. Según Chinchilla (2010) la familia del empleado es el nuevo stakeholder de la empresa y por eso tratar temas de equilibrio vida laboral-vida personal es parte de la responsabilidad social interna de las organizaciones. Ella menciona el término de «empresas familiarmente responsables».

Families and Work Institute realizó el estudio titulado Times are changing. Gender and Generation at work and at home (Galinsky, Aumann y Bond 2009) en él, se presentan actitudes actuales del hombre y la mujer en Estados Unidos sobre temas de igualdad de oportunidades. Por ejemplo, el porcentaje de empleados que en 2008 cree que las madres que trabajan pueden ser buenas madres y tener una relación con sus hijos como las madres que no trabajan es $73 \%$, mientras que en 1977 era solo $58 \%$. Es la opinión de los hombres la que más ha variado en este lapso de tiempo.

En el mismo estudio se señala que los hombres están pasando más tiempo con sus hijos menores de 13 años. Hace tres décadas eran 2 horas por día de trabajo y actualmente es 3 horas. Las madres siguen dedicándoles 3.8 horas en promedio a sus hijos. Los hombres también están asumiendo mayores responsabilidades en el cuidado de sus hijos en 2008 que en 1992. Lo mismo sucede en cuanto a las tareas del hogar, especialmente en lo que se refiere a cocinar. Los hombres en 2008 afirman que se encargan en un $56 \%$ de esta labor mientras que en 1992 solo un $34 \%$ lo hacía. Se desprende del estudio que es socialmente aceptable que los hombres estén y que digan que están involucrados con el cuidado de los hijos, la cocina y la limpieza.

Otro estudio de Families and Work Institute y The Center of Aging and Work sobre las experiencias de hombres y mujeres mayores de 50 años en el lugar de trabajo (Bond, Galinsky, Pitt-Catsouphes y Smyer 2005) presenta un índice para medir flexibilidad en el trabajo. Los elementos que se consideran para medir la flexibilidad en el trabajo que tienen los hombres y mujeres son los siguientes: Tener tiempo flexible periódicamente, tener tiempo flexible diariamente, tener aprobación para tomar tiempo dentro del horario de trabajo para ocuparse de asuntos familiares, tener la posibilidad de ausentarse del trabajo algunos días para hacerse cargo de alguien y poder trabajar algo desde casa, tener la posibilidad de tomar «tiempos de descanso» cuando se requiere, trabajar en el turno que se 
desea, tener completo o mucho control sobre el horario de trabajo, tener la posibilidad de trabajar a tiempo parcial (si se es trabajador a tiempo completo) y a tiempo completo (si se es trabajador a tiempo parcial), poder utilizar la flexibilidad de tiempo sin poner en peligro la línea de carrera en la empresa, poder trabajar una semana laboral comprimida y poder trabajar parte del año (cuando no es por causa de la estacionalidad).

Según Guillaume y Pochic (2009), las mujeres aplican ciertas estrategias para poder alcanzar posiciones de dirección. Cuando son muy jóvenes tratan de ser "las hijas» de algún gerente y acceder a viajes como expatriadas. Asimismo, unas cuantas optan por mantenerse solteras o sin niños al comienzo de su carrera. Una estadística interesante menciona que $30 \%$ de las mujeres ejecutivas viven solas (solteras o divorciadas), mientras solo el $6 \%$ de sus colegas hombres están en la misma situación. Sobre el hecho de trabajar largas horas las mujeres retrasan la maternidad y tratan de embarazarse cuando ya han alcanzado cierta estabilidad.

Es importante mencionar que el tiempo que dedican los hombres al trabajo remunerado determina el tiempo que le pueden dedicar a la familia, sin embargo, con las mujeres ocurre exactamente lo contrario (Padilla Lavín 2010). Padilla menciona que el tema de la maternidad hace que las mujeres sean consideradas incapaces temporalmente y que en algunos casos las limitaciones se extienden por varios años restringiendo las posibilidades de las mujeres de crecer laboralmente.

La literatura revisada menciona repetidamente algunos factores que afrontan las mujeres como impedimento para alcanzar los puestos más altos en las empresas. Las barreras que encuentran las mujeres para ascender, según un estudio titulado La mujer directiva en España: catalizadores e inhibidores en las decisiones de trayectoria profesional (De Anca y Aragón 2007) son: dificultad de conciliación entre la vida profesional y la vida privada, falta de flexibilidad en el entorno laboral, percepciones negativas que soporta la mujer directiva y falta de modelos femeninos como son las mentoras y las redes de apoyo.

A propósito de los estereotipos Un reciente estudio sobre el liderazgo en hombres y mujeres presenta información interesante (Prime, Carter y Welbourne 2009). Se pueden apreciar en la siguiente tabla los estereotipos relacionados a comportamientos de liderazgo en hombres y mujeres.

\section{Tabla № 1: Estereotipos relacionados a comportamientos de liderazgo}

\begin{tabular}{|c|c|}
\hline Comportamientos femeninos & Comportamientos masculinos \\
\hline Brindar soporte, ánimos y recursos a otros. & $\begin{array}{l}\text { Solucionar problemas. Actuar con decisión para acabar con } \\
\text { los obstáculos al desempeño. }\end{array}$ \\
\hline $\begin{array}{l}\text { Reconocer con incentivos monetarios y no monetarios cuando } \\
\text { sea necesario. }\end{array}$ & Influenciar a quienes ocupan posiciones superiores. \\
\hline Facilitar el desarrollo de los subordinados. & Delegar. Autorizar a otros para que tomen responsabilidades. \\
\hline $\begin{array}{l}\text { Desarrollar y mantener relaciones con otros que pueden } \\
\text { proveer recursos a la organización. }\end{array}$ & \\
\hline Consultar con otros antes de tomar decisiones. & \\
\hline $\begin{array}{l}\text { Fomentar el trabajo en equipo, el identificarse con la organi- } \\
\text { zación. }\end{array}$ & \\
\hline $\begin{array}{l}\text { Motivar a otros a tener mayor entusiasmo y compromiso } \\
\text { apelando a sus emociones o ejemplos personales. }\end{array}$ & \\
\hline
\end{tabular}


El mencionado estudio revela que muchas de las decisiones que se toman sobre quiénes ocuparán posiciones de liderazgo se basan en estos estereotipos. Es decir que se relaciona el sexo del líder con el desempeño del líder. Además, este estudio concluye que, aunque hoy se diga que los comportamientos de liderazgo femeninos son muy necesarios en las empresas, los hombres no reconocen que las mujeres destaquen en estos, por lo que aún está lejana la aceptación de líderes femeninos en el mundo corporativo.

\section{Metodología}

Se realizó un estudio empírico preliminar. Una primera etapa consistió en la revisión bibliográfica de documentos que se ocupan de la mujer en el mercado laboral latinoamericano.
La segunda etapa consistió en la elaboración de una guía de entrevistas para ejecutivas y otra para representantes de instituciones vinculadas con el tema. La tercera etapa consistió en la aplicación de las entrevistas y en la realización de un grupo de enfoque con ejecutivas de éxito. Se entrevistó 10 ejecutivas con experiencia de dirección en empresas que operan en el Perú y en Latinoamérica y 5 expertos en el tema. En esta etapa también se tuvo la oportunidad de realizar un de grupo de enfoque con 18 miembros de la Organization of Women in International Trade - OWIT. Las participantes fueron mujeres de éxito en los negocios en el Perú y estuvieron muy dispuestas a aportar su opinión sobre los tópicos de la investigación. La cuarta etapa consistió en el análisis de los resultados.

\section{Tabla № 2: Perfil de las ejecutivas exitosas entrevistadas}

\begin{tabular}{|c|c|c|c|c|}
\hline Entrevistada & Semblanza & Edad & Estado civil & $N^{\circ}$ de hijos \\
\hline Entrevistada 1 & $\begin{array}{l}\text { Ejecutiva perteneciente al sector de consultoría y gobernanza. } \\
\text { Estudió la carrera de administración y posteriormente la } \\
\text { de ingeniería de sistemas. Ha desempeñado posiciones de } \\
\text { liderazgo de una importante empresa a nivel nacional y regional. } \\
\text { Actualmente es miembro de directorios de diversas empresas. }\end{array}$ & 52 & Casada & 3 \\
\hline Entrevistada 2 & $\begin{array}{l}\text { Ingeniera industrial de profesión. Inició su carrera laboral como } \\
\text { asistente de la gerencia financiera, pasó al gerenciamiento } \\
\text { de una agencia de aduanas y actualmente es Gerente de } \\
\text { Administración y Finanzas de una importante empresa en la } \\
\text { industria para la panificación. }\end{array}$ & 44 & Casada & 1 \\
\hline Entrevistada 3 & $\begin{array}{l}\text { Ingeniera Industrial de profesión. Empezó a trabajar desde los } \\
20 \text { años en una gran empresa que le permitió un desarrollo de } \\
\text { largo plazo y estar en constantes capacitaciones. Actualmente, } \\
\text { es consultora independiente y docente. }\end{array}$ & 39 & Casada & 1 \\
\hline Entrevistada 4 & $\begin{array}{l}\text { Administradora de profesión. Por mucho tiempo estuvo a cargo } \\
\text { de los negocios familiares, por lo que desde muy joven comenzó } \\
\text { a trabajar. Debido a los negocios que lideraba estudió también } \\
\text { para cheff profesional. Actualmente dirige un programa de } \\
\text { gastronomía en importante instituto del sector. }\end{array}$ & 49 & Casada & 3 \\
\hline Entrevistada 5 & $\begin{array}{l}\text { Estudió la carrera de contabilidad e informática. Comenzó } \\
\text { su carrera laboral en una comercializadora de arroz, pasó a } \\
\text { trabajar para el Estado, para el rubro de gaming, entre otros. } \\
\text { Actualmente se desempeña como Gerente de Administración y } \\
\text { Finanzas de una importante empresa en el sector de energía. }\end{array}$ & 42 & Soltera & 0 \\
\hline
\end{tabular}




\begin{tabular}{|l|l|c|c|c|}
\hline Entrevistada & Semblanza & Estado civil & $N^{\circ}$ de hijos \\
\hline Entrevistada 6 & $\begin{array}{l}\text { Estudió la carrera de ingeniería industrial. Al terminar la } \\
\text { universidad trabajó para una gran empresa donde pasó } \\
\text { varios años, desempeñando distintos puestos. Actualmente se } \\
\text { desempeña como Gerente del área de Mantenimiento y Servicios } \\
\text { Generales de una prestigiosa institución. }\end{array}$ & Casada & 1 \\
\hline Entrevistada 7 & $\begin{array}{l}\text { Estudió la carrera de contabilidad. Se desenvuelve tanto en la } \\
\text { parte tributaria como financiera de su carrera. Ha desempeñado } \\
\text { funciones para el sector público y actualmente se encuentra } \\
\text { trabajando para el sector privado. Ha alcanzado cargos de } \\
\text { supervisiones y jefaturas. Además de trabajar para una importante } \\
\text { entidad financiera en el cargo de Gerente de Administración y } \\
\text { Finanzas, también labora de manera independiente. }\end{array}$ & Soltera & 0 \\
\hline Entrevistada 8 8 & $\begin{array}{l}\text { Comunicadora de profesión. Lleva 25 años trabajando en el } \\
\text { rubro de cosmética. Llegó a ser gerente de línea a los 30 años } \\
\text { de edad. Actualmente es directora de división en el Perú de una } \\
\text { reconocida marca internacional del sector. }\end{array}$ & 50 & Casada & 2 \\
\hline Entrevistada 9 9 & $\begin{array}{l}\text { Médica pediatra de profesión. Ha trabajado en organizaciones } \\
\text { sin fines de lucro; tanto para instituciones del Estado como para } \\
\text { ONG's en cargos directivos. }\end{array}$ & 56 & $\begin{array}{l}\text { Casada } \\
\text { Inicío su carrera estudiando secretariado, y posteriormente } \\
\text { trabajó en una entidad financiera, donde a la vez inicio sus } \\
\text { estudios de administración. Fue propietaria de una casa de } \\
\text { cambios y de esta manera empezó una fructífera carrera } \\
\text { como empresaria. Hoy es dueña de un prestigioso centro de } \\
\text { entretenimiento. }\end{array}$ & 3 \\
\hline Entrevistada 10 & Casada & 2 \\
\hline
\end{tabular}

Elaboración propia.

Para efectos de este estudio el concepto de éxito se relaciona con haber alcanzado cargos de gerencias de alto nivel en áreas tradicionalmente manejadas por hombres y en organizaciones de reconocido prestigio. Por ello, las diez entrevistadas se consideran mujeres ejecutivas exitosas.

Los representantes de instituciones y los expertos en temas de mujeres ejecutivas y emprendedoras entrevistados son comunicadores, periodistas, representantes de asociaciones de mujeres y del Ministerio de la Mujer.

Las entrevistas fueron transcritas y luego se procedió a su codificación. Para brindarle confiabilidad al procedimiento se procedió a comparar codificaciones entre el investigador principal y sus asistentes de investigación.

\section{Resultados}

Debido a la naturaleza del estudio los resultados son cualitativos. Se mostrarán resultados sobre las estrategias que aplican las mujeres ejecutivas que han logrado posiciones de dirección. Algunos de los testimonios de las entrevistadas ilustrarán los resultados mostrados.

\section{Las estrategias}

Esfuerzo-Perseverancia. La primera de las estrategias que sale a relucir es la del esfuerzo que la mujer tiene que realizar frente a sus pares masculinos. Lo que las entrevistadas reflejan es que ellas son conscientes de que tienen que sobresalir y esto se logra «dando más del $100 \%$ ». Las ejecutivas que han llegado a ocupar las más altas posiciones en sus empresas son muy responsables, muy trabajadoras y buscan la excelencia en lo que realizan. 


\section{Testimonios}

\begin{tabular}{|l|l|} 
& $\begin{array}{l}\text { «Dónde hay una mayor predominancia } \\
\text { de personal masculino, sí hay un mayor } \\
\text { tema de esfuerzo. Un hombre se esfuerza } \\
1,2,3, \text { una mujer tiene que esforzarse } \\
4,5, \text { más, el doble. Pero eso es por un } \\
\text { tema de feeling, la cultura, un tema de } \\
\text { comunicación, estilos de comunicación». }\end{array}$ \\
Esfuerzo - & $\begin{array}{l}\text { "Creen que la mujer es handicap pero la } \\
\text { mujer siempre tiene que estar demostrando } \\
\text { que no es así. La mujer tiene que trabajar } \\
\text { mejor para dar el ejemplo». }\end{array}$ \\
\hline $\begin{array}{l}\text { "El éxito lo debo a la perseverancia. } \\
\text { Destacaba porque hacía más que los } \\
\text { demás». }\end{array}$
\end{tabular}

Objetivos claros. Adicionalmente, las entrevistadas resaltan y recomiendan el fijarse objetivos claros. Es importante crear una visión de la profesional que se desea llegar a ser. En relación a este punto, en diferentes momentos de las entrevistas mencionaron la actitud positiva, el saber perseverar a pesar de que las circunstancias no se presenten como se esperaban. Es a raíz de que los obstáculos se pueden presentar de distintas formas que los objetivos fijados pueden brindar claridad.

\section{Testimonio}

$\begin{array}{ll} & \text { "Tú puedes tener tu plan, pero el plan } \\ \text { nunca se lleva a cabo tal cual lo has } \\ \text { diseñado. Entonces en el camino las } \\ \text { Objetivos } \\ \text { claros }\end{array}$

Preparación. Para las ejecutivas entrevistadas no basta con estudiar una carrera, se han dado el tiempo de especializarse y de actualizarse permanentemente. Algunas, por diferentes factores, no habían podido estudiar una maestría, y a pesar de que ya han alcanzado sus metas profesionales decidieron darse la oportunidad de estudiarla en su madurez.

\section{Testimonios}

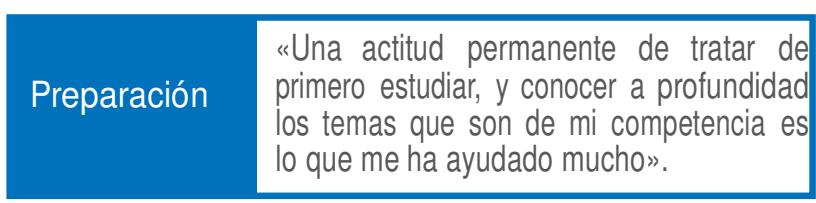

Apoyo en casa. El poder contar con apoyo en casa es importantísimo. Alguna se refirió al «ejercito» que le brindaba soporte en casa. Resaltamos entonces el apoyo familiar como impulso para poder dedicarle tiempo y esfuerzo al desarrollo de una carrera profesional.

\section{Testimonio}

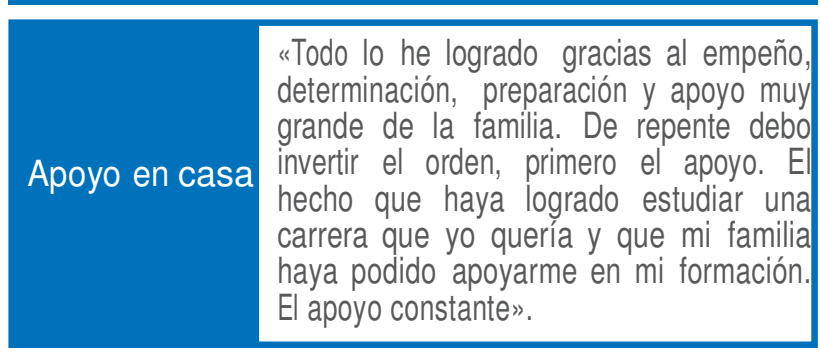

Equilibrio vida personal-laboral. Las ejecutivas entrevistadas mencionan como estrategia la flexibilidad tanto en los horarios de trabajo como en la posibilidad de trabajar desde casa. Todas las ejecutivas entrevistadas están trabajando en la conciliación de temas laborales y personales, no solamente por su familia sino para lograr tiempo para ellas. Dentro de este ámbito se reservan vacaciones para disfrutarlas con la familia e involucran a la familia con sus objetivos profesionales haciéndola partícipe de

\section{Testimonios}

\begin{tabular}{|c|c|}
\hline \multirow{2}{*}{$\begin{array}{l}\text { Equilibrio } \\
\text { entre la vida } \\
\text { y el trabajo }\end{array}$} & $\begin{array}{l}\text { «He tenido que sacrificar, temas } \\
\text { personales, gustos en realidad. Tiempo } \\
\text { para hacer actividades como arte, } \\
\text { deportes, me encanta hacer actividades } \\
\text { manuales, me encanta cocinar, pero esas } \\
\text { son las actividades que dejo de hacer». }\end{array}$ \\
\hline & $\begin{array}{l}\text { «o que siempre me han reclamado es } \\
\text { tiempo, y yo nunca he tenido tiempo. } \\
\text { ¿Qué hay que hacer? El tiempo no es solo } \\
\text { para ti, es también para compartir con la } \\
\text { familia, con la pareja. Y lo que he hecho } \\
\text { es administrar mi tiempo, el administrar } \\
\text { mi tiempo ya me permite hacer todo». }\end{array}$ \\
\hline
\end{tabular}


Adaptación- Masculinización. La estrategia que se trata de explicar bajo este título es de las que más se mencionó, no solo ante la pregunta sobre las estrategias sino a lo largo del resto de la entrevista. Las entrevistadas reconocen que tuvieron que adaptarse a los estilos de las empresas y se utiliza el término «masculinización» porque ellas mismas refieren que los hombres se desenvuelven de determinada manera y que ellas tienen que hacer lo posible por imitarlos. Uno de los temas que más se ha mencionado es el de la comunicación, en lo referente a ser directos, y a trabajar en un posicionamiento personal. Otro factor es el tener que ser un poco más agresivo de lo normal, el ser menos sensible o incluso dejar de lado que se es mujer para convertirse en un profesional, simplemente, sin importar el sexo.

\section{Testimonios}

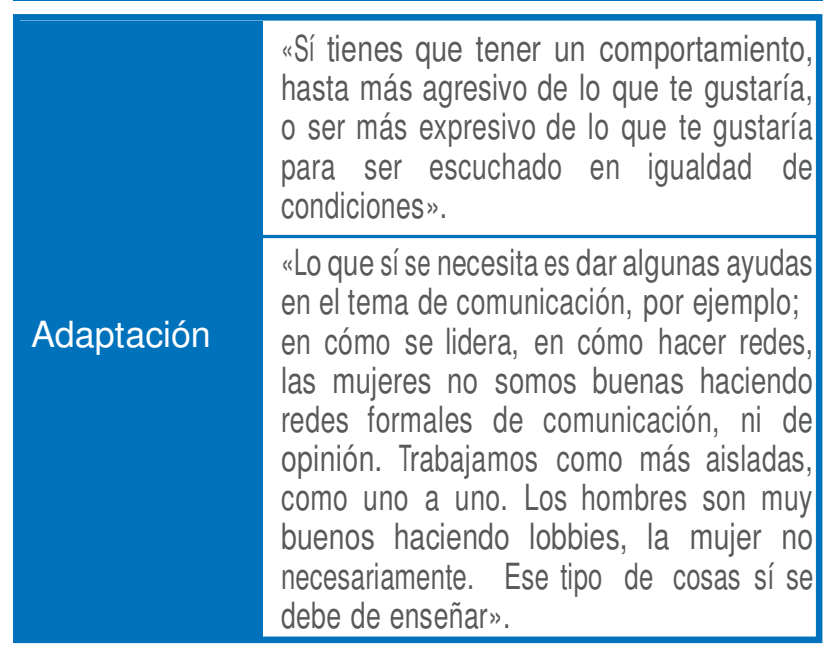

El factor de perder la esencia femenina y adoptar posturas un tanto masculinas en el ámbito empresarial es un comportamiento que no llama la atención a las entrevistadas que lo han mencionado. Forma parte del día a día que enfrentan, sin embargo muestra que no son las organizaciones las que se adaptan a sus miembros, sean hombres o mujeres, sino por el contrario son las personas las que deben cambiar, aunque esto sea en temas tan privados como la manera de ser de cada uno.

Algunas estrategias que las ejecutivas entrevistadas no han planteado directamente ante la pregunta de las medidas que aplicaron para alcanzar su objetivo, pero que sin embargo, fueron mencionadas en otros momentos de la entrevista fueron: el tomar conciencia del marketing personal, el concentrar los viajes al inicio de la carrera y el retrasar la maternidad.

\section{Conclusiones}

Los entrevistados han brindado testimonios valiosos sobre la situación compleja que afronta la mujer cuando desea ocupar cargos de dirección y los caminos para lograrlo.

Las ejecutivas exitosas entrevistadas se han preparado para alcanzar sus posiciones. Afrontan la doble jornada laboral pero cuentan con amplio apoyo en casa, sea de empleadas o de familiares, por lo que pueden enfocarse más en actividades laborales que domésticas. En este sentido su dedicación al trabajo se equipara con la de sus colegas varones.

Las estrategias que han seguido son: a) ser perseverantes a lo largo de su trayectoria laboral a pesar de episodios de desigualdad de oportunidades que les ha tocado vivir; b) definir objetivos claramente; c) prepararse académicamente y actualizarse permanentemente; d) contar con apoyo para manejar asuntos domésticos; e) aprovechar las posibilidades de trabajo flexible y equilibrar asuntos laborales y personales; y f) adaptarse a los estilos de los negocios en los que participaron.

El estudio realizado es exploratorio y como tal enfrenta diversas limitaciones. Una de las limitaciones es el tamaño de la muestra. Hubiera sido deseable entrevistar a un mayor número de mujeres profesionales exitosas, de manera que se ilustraran mejor los resultados obtenidos. Adicionalmente, una muestra mayor probablemente hubiera contribuido en la identificación de factores adicionales que contribuyan o inhiban el desarrollo profesional de la mujer ejecutiva.

Las empresas deben comprender que tanto hombres como mujeres tienen que ser ayudados para tener éxito en el trabajo y en el hogar. El desequilibrio trae consecuencias negativas a la empresa. 
Es oportuno preguntarse si se están dando las facilidades en las empresas para apoyar a hombres y mujeres que tienen responsabilidades familiares y que desean progresar en su carrera. Además las empresas deben hacer un esfuerzo por conocer qué motiva a los hombres y mujeres de diferentes generaciones, para proveerles del lugar de trabajo que los retenga.
La reflexión final se orienta a promover estudios específicos de la mujer en la realidad empresarial latinoamericana con miras a aportar propuestas que faciliten que la mujer logre posiciones importantes en la empresa. 


\section{BIBLIOGRAFÍA}

ALLES, M.

2000 Mujeres, trabajo y autoempleo. Otro camino frente al desempleo y desarrollo de carrera. Buenos Aires: Granica.

ANZORENA, C.

2008 «Estado y división sexual del trabajo: las relaciones de género en las nuevas condiciones del mercado laboral». Utopía y Praxis Latinoamericana, pp. 47-68.

BOND, J., E. Galinsky, M. Pitt-Catsouphes y M. Smyer 2005 Families and Work Institute. Noviembre de 2005, recuperado el 09 de junio de 2010, de http://www.familiesandwork.org/.

\section{CATALYST}

2009 Women Leaving and Re-entering the Workforce.Recuperado el 11 de junio de 2010, de Catalyst: http://www.catalyst.org/page/64/ browse-research-knowledge.

CHINCHILLA, N.

2010 «Conciliación de la vida laboral, familiar y personal». 19 Congreso peruano de Gestión de Personas. Lima, 12 de octubre de 2010.

DAEREN, L.

2000 «Mujeres empresarias en América Latina: El difícil equilibrio entre dos mundos de trabajo. Desafíos para el futuro». Primer Seminario Internacional de la Mujer Empresaria, pp. 2-13. Santiago de Chile.

DE ANCA, C. y S. Aragón

2007 «La Mujer directiva en España: catalizadores e inhibidores en las decisiones de trayectoria profesional». Academia Revista Latinoamericana de Administración, 45-63.

DEBELJUH, P.

2010 Work and Family: «A view from Latin America». En N. Chinchilla, M. Las Heras, \& A. D. Masuda, Balancing Work and Family, pp. 5978. Massachusetts: HRD Press, Inc.

FOMIN

2010 Dueña de tu propia empresa: Identificación, análisis y superación de las limitaciones a las pequeñas empresas de las mujeres en América Latina y el Caribe. Washington: Banco Interamericano de Desarrollo.

FORTUNE

2010 CNN Money. 3 de mayo de 2010, recuperado el 13 de agosto de 2010, de http://money. cnn.com/magazines/fortune/fortune500/2010/ womenceos/.

GALINSKY, E., K. Aumann y J. Bond

2009 Families and Work Institute. Recuperado el 11 de Junio de 2010, de http://www. familiesandwork.org/.

GARCÍA, M.

2010 «Tierra de Hombres». América Economía, pp. 52-53.

GTZ, Banco Mundial y BID

2010 Mujeres Empresarias: Barreras y Oportunidades en el sector Privado Formal en América latina. Washington: Banco Mundial.

GUILLAUME, C. y S. Pochic

2009 «What would you sacrifice? Access to Top Management and the Work - Life Balance». Gender, Work and Organization, pp. 14-34.

NEWELL, S

2002 «La discriminación en el trabajo». En S. Newell, Creando organizaciones saludables- bienestar, diversidad y ética en el trabajo. Thomson.

OIT

2010 Women in Labour markets: measuring progress and idenifying challenges.Ginebra: OIT.

2007 La igualdad en el trabajo: afrontar los retos que se plantean. Recuperado el 07 de mayo de 2010, de www.ilo.org/declaration.

PADILLA LAVÍN, M. D.

2010 «En torno a la incorporación de la mujer al mercado laboral y su impacto en la esfera privada». Hospitalidad- ESDAI, pp. 97-126.

PRIME, J., N. Carter, y T. Welbourne

2009 «Women "Take care", Men "Take charge": Managers' Stereotypic Perceptions of Women and Men Leaders». The Psychologist-Manager Jornal, pp. 25-49.

THORNTON, G.

2012 Mujeres en altos cargos gerenciales aún no son muchas - Grant Thornton International Business Report 2012 . Grant Thornton International Ltd.

WORLD ECONOMIC FORUM

2010 The Global Gender Gap Report. Retrieved Noviembre 2010, from http://www. weforum.org/en/Communities/Women\%20 Leaders\%20 and \%20Gender\%20Parity/ GenderGapNetwork/index.htm. 